\title{
The evaluation of different smile parameters in the Turkish population
}

\author{
Merve Köseoğlu1 ${ }^{1}$, Hatice Özdemir' ${ }^{\oplus}$, Funda Bayındır \\ ${ }^{1}$ Ataturk University, Faculty of Dentistry, Department of Prosthodontics, Erzurum, Turkey
}

\section{Correspondence:}

\section{Dr. Hatice ÖZDEMIR}

Ataturk University, Faculty of

Dentistry, Department of

Prosthodontics, Erzurum, TURKEY.

E-mail:dentist_hatice@hotmail.com

Received: 16 January 2018

Accepted: 24 February 2018

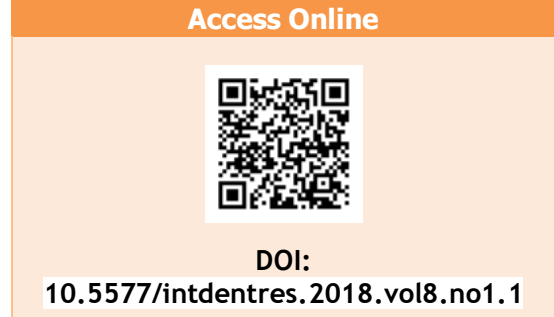

\section{Introduction}

Nowadays, the number of patients who consult dentists to achieve a perfect smile is gradually increasing. In the past, patients and dentists thought

\begin{abstract}
Aim: In the past, patients and dentists thought that the dental aesthetics could only be ensured with modifications on teeth. Together with the latest developments in the smile analysis and aesthetic design, researchers have identified the aesthetics based on facial, oral-facial, dentogingival, and dental components. The aim of this study is to evaluate the smile type (ST), smile line (SL), maxillary incisal line types (ML), and buccal corridor widths $(B C)$ related to the smile aesthetics.

Methodology: A total of 190 individuals (92 males and 98 females) aged between 18 and 25 years, living in different cities of Turkey and studying at Atatürk University, Faculty of Dentistry were included in the study. In the study, the smile types, smile lines, maxillary incisal curvatures and buccal corridor widths of the individuals were examined.

Results: In the young Turkish population, the most common ST in both genders is the commissural smile, and the least common ST is the complex smile. The most common SL is the SL, and the least common $\mathrm{SL}$ is the high $\mathrm{SL}$, and it varies by gender. While the most common ML is the convex line in both genders, the least common $M L$ is the reverse incisal line. While the last visible maxillary tooth during the social smile is mostly the maxillary second premolar tooth, it is the maxillary second molar tooth at least.

Conclusions: The incidence of some smile parameters in young Turkish population varies according to gender.
\end{abstract}

Keywords: Smile type, smile line, maxillary anterior incisal curve, buccal corridor width

How to cite this article: Köseoğlu $M$, Özdemir $H$, Bayındır $F$. The evaluation of different smile parameters in the Turkish population. Int Dent Res 2018;8(1):1-6. that the dental aesthetics could only be ensured with modifications on teeth (1). Clinicians following current developments have accepted the necessity of evaluating the smile components to provide the optimal dental aesthetics (2). In order to achieve 
success in aesthetic dentistry, patients should not be applied a uniform standardized smile, each patient should be evaluated as a separate subject, their needs and expectations should be considered. To achieve an ideal smile, a combination of scientific aesthetic principles and artistic creativity is required (3). Traditionally, dental and facial aesthetics are defined by the macro and microelements. While the macroaesthetics examines the relationship between the face, lips, gums, and teeth, the micro-aesthetics examines the color and form of teeth (4). Together with the latest developments in the smile analysis and aesthetic design, researchers have identified the aesthetics based on facial, oral-facial, dentogingival, and dental components $(5,6)$. The relationship between the total facial form and balance is examined while evaluating the facial aesthetics, the relationship between the face, maxilla, and mandible and the relationship of the dental midline with the face is examined while evaluating the oral-facial aesthetics, the relationship of the lips with the teeth arches, teeth, and gums is examined while evaluating the oral aesthetics, the relationship between the teeth and gums is examined while evaluating the dentogingival aesthetics, and the macro and micro dental aesthetic parameters among the teeth and within each tooth are examined while evaluating the dental aesthetics (4).

Patients' smiles may be in different formations from each other. Researchers have noted that there are generally three different styles of the smile in the population. The first one of these is the commissural smile when the corners of the mouth are curled up by the zygomaticus major and the lips are seen in a diamond shape. The second one is the cuspid smile when the corners of the mouth are not curled up and the upper lip is elevated. The third one is the complex smile when the lower lip moves down without the corner of the upper lip mouth curling up (7).

One of the structures to be evaluated when analyzing the patients' smile is the lips that form the frame of a smile and thus define the aesthetic area (3). 2 types of smiling occur when the lips are functioning. The social smile is the type of smile that is voluntary, continuously repetitive, and not related to emotions. The spontaneous smile is the smile that develops depending on emotions, involuntarily, and is characterized by much bigger facial movements such as the squinting of the eyes and a maximum elevation of the lips compared to the social smile (2). The social smile has gained importance in dentistry studies since it can be repeated over time (8). The amount of teeth shown during a smile can be classified as high, medium, and low. However, this classification is not very clear (9). The low SL is described as the case in which the upper lip extends from the half of the crown lengths of incisors to the three incisors; the medium $\mathrm{SL}$ is described as the case in which 1-2 mm of gums are visible with teeth; and the high SL is described as the case in which a large part of the gums is visible together with teeth (3). However, the amount of the soft tissue shown during smiling is not the most critical aesthetic parameter (10). The smile symmetry is necessary to ensure an aesthetic smile. For the optimal smile symmetry, the commissural line and the occlusal line should overlap with the interpupillary line. The symmetrical image of the right and left sides of the middle line provides patients with an attractive smile (3).

The smile arch is defined as the relationship of the curvature of the incisal edges of the maxillary incisors with the curvature of the lower lips during a social smile (11). In an aesthetic smile, the incisal edge line should follow and be parallel to the curvature of the lower lip. In some cases, the incisal edge may be flat or reverse. The incisal plane must be vertical to the facial and dental midline in order to achieve symmetry in the smile (3).

The buccal corridor defines the area between the buccal surfaces of the posterior teeth and the commissures during a smile. The last visible maxillary teeth during a social smile determine the width of the buccal corridors. In an ideal smile, bilateral spaces should be symmetrical and as small as possible $(11,12)$.

In this study, it has been aimed to evaluate the ST, $\mathrm{SL}, \mathrm{ML}$ types, and $\mathrm{BC}$ related to the smile aesthetics that has not been evaluated until this time in the Turkish population. It is thought that these parameters will be effective in determining the relationship of teeth with the surrounding tissues after the correct determination of the tooth size, especially in totally edentulous patients. The present study hypothesis is that factors that have a significant effect on the smile aesthetics will vary by genders.

\section{Materials and Methods}

A total of 190 subjects ( 92 males and 98 females) aged between 18 and 25 years, living in different cities of Turkey and studying at Atatürk University, Faculty of Dentistry were included in the study. The study protocol was approved by the Institute's Ethics Committee and conducted in accordance with the principles of the Declaration of Helsinki.

The criteria required for students to be included in the study are as follows:

1. Not having a missing maxillary and mandibular anterior tooth, crowding, or diastema

2. Not having gingival or periodontal problems disrupting the relationship between teeth and surrounding tissues 
3. Not having a decay, restoration, abrasion, and fracture in maxillary and mandibular anterior teeth

4. Not having a history of orthodontic treatment

5. Not having dentofacial deformities and facial asymmetry

In order to achieve standardization in photography, the heads of the subjects were fixed by the forehead and gonion in the panoramic $\mathrm{X}$-ray device. Thus, it was ensured that the Frankfort horizontal plane was parallel to the floor (Fig. 1). Photographs were taken frontally using a DSLR camera (Nikon, DX SWM VR ED IF Aspherical $\varnothing 72$, Thailand) with a Nikon AF-S DX18-200/3.5-5.6 lens. The photographs were taken by the same person, always at the same distance, and in a way that the midline of the face was in the middle of the camera lens during social smiles of the subjects. The settings of the camera were kept fixed while taking photographs. In the study, it was examined which type of smile the volunteers had among the commissural, cuspid, and complex types (Fig. 2). In this study, the SL of the subjects was examined, and the SLs of the subjects were classified as low, medium, and high (Fig. 3).

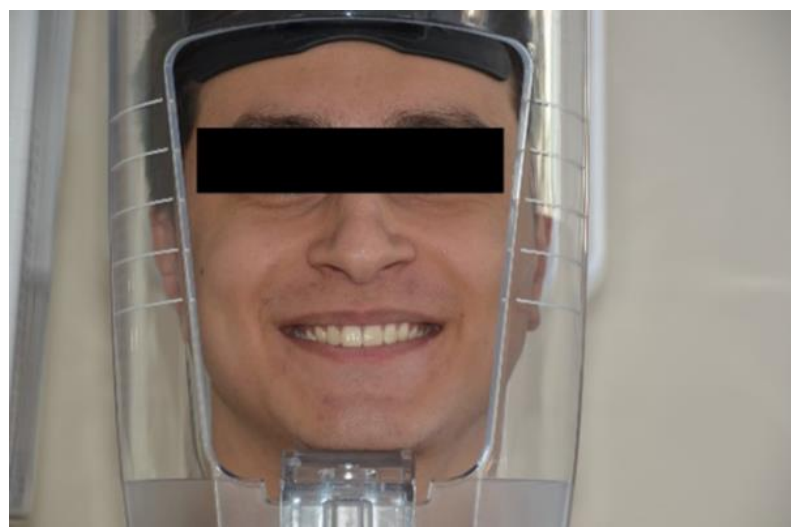

Figure 1. The immobilization of subject's head on panoramic X-ray device

The MLs of the volunteers were examined in 3 separate ways as convex, straight, and concave (Fig. 4). In the present study, the last visible maxillary teeth of the volunteers were examined during the social smile, and an idea was obtained indirectly about the BC of each subject.

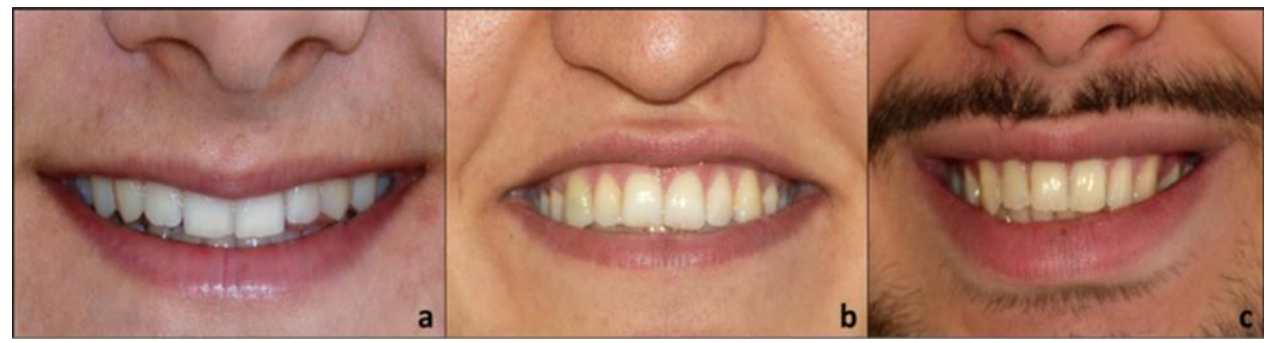

Figure 2. Smile types; a) Commissural smile, b) Cuspid smile, c) Complex smile

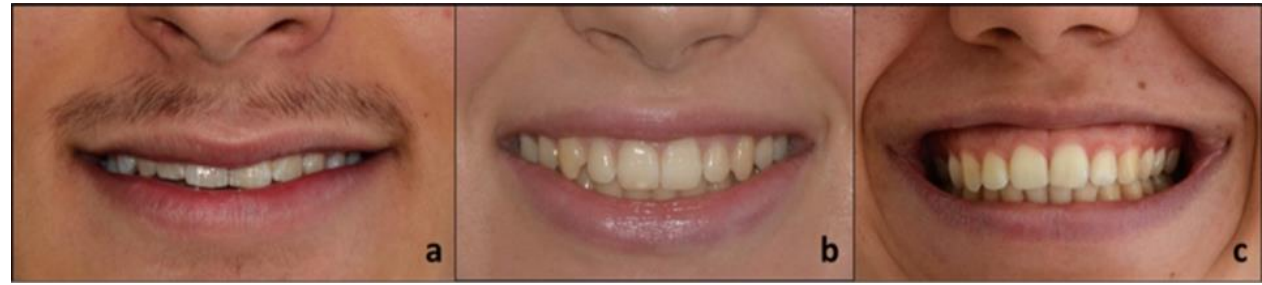

Figure 3. Smile line types; a) Low smile line, b) Medium smile line, c) High smile line

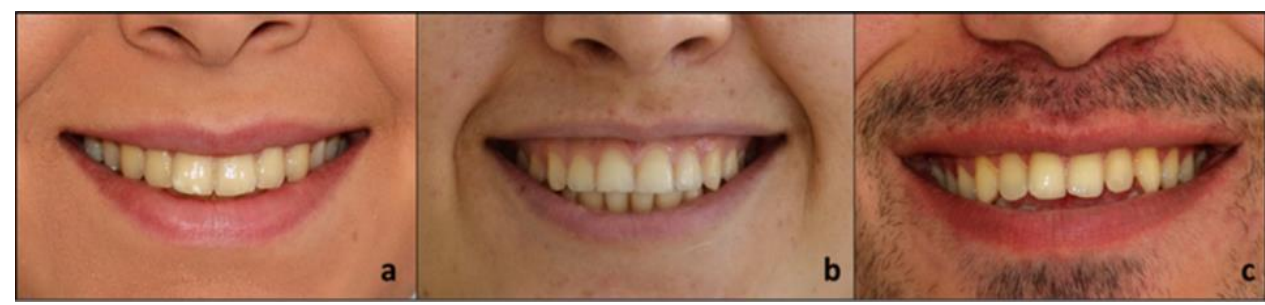

Figure 4. Maxillary incisal line types; a) Convex incisal line, b) Straight incisal line, c) Concave incisal line

It was examined which teeth among the maxillary canine, maxillary first premolar, maxillary second premolar, maxillary first molar, and maxillary second molar teeth were visible while the subjects were smiling voluntarily (Fig. 5). 


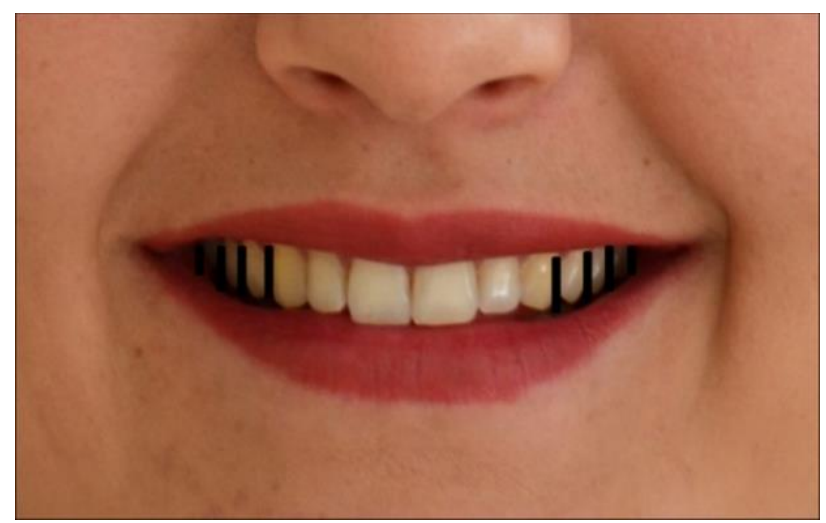

Figure 5. Schematic illustration of buccal corridor

The photo images obtained were transferred to a personal computer and examined in a suitable software program (Microsoft Office Picture Manager). The ST, $\mathrm{SL}$, smile arch, and BC of the subjects were examined by a single researcher, and the obtained data were transferred to the computer environment.

\section{Statistical Analysis}

The descriptive statistics of the obtained data of different smile parameters were performed by using the SPSS package program. Furthermore, gender differences were analyzed by the Chi-square test.

\section{Results}

Upon examining the ST of the volunteers in the study, it was determined that while the most common ST in both genders was the commissural ST, the least common ST was the complex ST (Table 1).

Table 1. Numbers (percentages) of incidence of the smiles types in the population

\begin{tabular}{lcccc} 
& Comissural (\%) & Cuspid (\%) & Compleks (\%) & Chi-square \\
\hline Female & $42(42,9)$ & $41(41,8)$ & $15(15,3)$ & \\
\hline Male & $41(44,6)$ & $27(29,3)$ & $24(26,1)$ & 4,787 \\
\hline Total & $83(43,7)$ & $68(35,8)$ & $39(20,5)$ & $(\mathrm{p}=0,091)$ \\
\hline
\end{tabular}

Table 2. Numbers (percentages) of incidence of the smile lines in the population

\begin{tabular}{|c|c|c|c|c|}
\hline & $\begin{array}{l}\text { Low smile } \\
\text { line (\%) }\end{array}$ & $\begin{array}{l}\text { Medium smile } \\
\text { line (\%) }\end{array}$ & $\begin{array}{c}\text { High smile line } \\
(\%)\end{array}$ & Chi-Square \\
\hline Female & $29(29,6)$ & $49(50,0)$ & $20(20,4)$ & \multirow{3}{*}{$\begin{array}{c}3,957 \\
(p=0,138)\end{array}$} \\
\hline Male & $40(43,5)$ & $37(40,2)$ & $15(16,3)$ & \\
\hline Total & $69(36,3)$ & $86(45,3)$ & $35(18,4)$ & \\
\hline
\end{tabular}

Table 3. Numbers (percentages) of incidence of the incisal line types in the population

\begin{tabular}{ccccc} 
& Convex (\%) & Straight $(\%)$ & Concave $(\%)$ & Chi-square \\
\hline Female & $71(72,4)$ & $22(22,4)$ & $5(5,1)$ & \\
\hline Male & $50(54,3)$ & $328(0,4)$ & $14(15,2)$ & $\begin{array}{c}8,447 \\
(p=0,015)\end{array}$ \\
\hline Total & $121(63,7)$ & $50(26,3)$ & $19(10,0)$ & \\
\hline
\end{tabular}

Table 4. Numbers (percentages) of incidence of the last visible maxillary teeth during a smile in the population

\begin{tabular}{|c|c|c|c|c|c|c|}
\hline & $\begin{array}{l}\text { Maxillary } \\
\text { Canin (\%) }\end{array}$ & $\begin{array}{l}\text { Maxillary First } \\
\text { Premolar (\%) }\end{array}$ & $\begin{array}{l}\text { Maxillary Second } \\
\text { Premolar (\%) }\end{array}$ & $\begin{array}{l}\text { Maxillary First } \\
\text { Molar (\%) }\end{array}$ & $\begin{array}{l}\text { Maxillary Second } \\
\text { Molar (\%) }\end{array}$ & Chi-square \\
\hline Female & $4(4,1)$ & $28(28,6)$ & $36(36,7)$ & $29(29,6)$ & $1(1,0)$ & \multirow{3}{*}{$\begin{array}{c}2,634 \\
(p=0,621)\end{array}$} \\
\hline Male & $3(3,3)$ & $34(37,0)$ & $35(38,0)$ & $19(20,7)$ & $1(1,1)$ & \\
\hline Total & $7(3,7)$ & $62(32,6)$ & $71(37,4)$ & $48(25,3)$ & $2(1,1)$ & \\
\hline
\end{tabular}

Significant differences between first- and secondyear students were detected in family histories of HBV positivity $(13.2 \%$ vs. $24.5 \%)$, serological testing $(11.4 \%$ vs. $30.6 \%)$, HBV carrier status (14\% vs. $56.9 \%)$, HBV immunization ( $36.7 \%$ vs. $47.7 \%)$, and development of a protective response $(3.2 \%$ vs. $19 \%$; all $\mathrm{p}<0.05)$. No 
significant relationship was observed between students' year in school and responses to other items (all p>0.05; Table 2).

No significant relationship was detected between survey responses and parents' educational levels or the presence of a healthcare worker in the family (both p>0.05; Tables 3, 4 and 5)

\section{Discussion}

In the present study, the STs, SLs, incisal line types, and $B C$ of the subjects were investigated in the young Turkish population. The results obtained from the study partially support study hypothesis. While there is no difference between the genders in terms of the ST and $\mathrm{ML}$, the SL and buccal corridor width vary by gender. Among the STs, the commissural smile is the most common smile in both genders, and the complex smile is the least common one. In terms of the ML, the most common one is the convex incisal line, and the least common one is the reverse incisal line. In the SL evaluation, while the low SL is the most common one in males and the medium SL is the most common one in females, the high $S L$ is the least common SL in both genders. In the $\mathrm{BC}$, while the most visible tooth in both genders is the maxillary second premolar, the order of visibility of other teeth varies.

The results of the studies conducted on subjects with natural teeth in a population may be a guidance for dentists when applying a prosthetic treatment to subjects in the same population. Especially, in the case of total edentulism or during the application of a frontal fixed prosthetic restoration, the parameters determined can provide convenience to the dentist. It is very important that especially the anterior teeth are in harmony with the surrounding tissues during a smile. These parameters determined as a result of the study can facilitate the construction of aesthetic restorations compatible with the surrounding tissues around the patient's mouth.

According to Rubin, while the most common ST in the American population is the commissural smile, the least common ST is the complex smile (13). $67 \%$ of the population have the commissural smile, $31 \%$ have the cuspid smile, and $2 \%$ have the complex smile. The results obtained from the present study are parallel to the results of the study of Rubin (13). According to this, the most common ST in the young Turkish population is the commissural smile $(43.7 \%)$, the least common ST is the complex smile (20.5\%). The cuspid smile is observed at the rate of $35.8 \%$.

In their studies conducted in the Saudi Arabian population, Al-Johany et al. determined that $80 \%$ of the population had the medium SL and $20 \%$ had the high SL
(14). However, Al-Johany et al. did not find the low SL in the population (14). According to Yoon et al. 55.83\% of the Korean population has the medium SL, 29.17\% have the high SL, and 15\% have the low SL (15). In the study they conducted, AlQahtani et al. determined that the medium SL was observed in $57.5 \%$ of the Saudi Arabian population, the high SL was observed in $24.1 \%$, and the low SL was observed in $18.4 \%$ (16). According to the results obtained from the present study, the most common SL in the young Turkish population is the medium SL (45.3\%), and the least common SL is the high SL (18.4\%). The low SL is observed at the rate of $36.3 \%$.

Al-Johany et al. determined in their studies that the convex incisal line was observed in $78 \%$ of the Saudi Arabian population and the straight incisal line was observed in $22 \%$ (14). However, Al-Johany et al. did not find a reverse incisal line in the population (14). In the study conducted in the Korean population, Yoon et al. reported that the convex incisal line was observed in $60.42 \%$ of the population, the straight incisal line was observed in $34.17 \%$, and the reverse incisal line was observed in $5.41 \%$ (15). According to the present study, while the most common incisal line type is the convex line type $(63.7 \%)$, the least common incisal line type is the reverse incisal line (10\%). The straight incisal line is observed at the rate of $26.3 \%$.

In the study they conducted, Al-Johany et al. reported that the last visible maxillary tooth during a smile was the maxillary first premolar tooth in $8 \%$ of the Saudi Arabian population, the maxillary second premolar tooth in $60 \%$, and the maxillary first molar tooth in $32 \%$ (14). Yoon et al. determined that the last visible maxillary tooth during a smile was the canine tooth in $0.84 \%$ of the Korean population, the maxillary first premolar tooth in $19.17 \%$, the maxillary second premolar tooth in $57.92 \%$, the maxillary first molar tooth in $20 \%$, and the maxillary second molar tooth in $2.08 \%$ (15). According to the present study, it is observed that the last visible maxillary tooth during a smile is the maxillary canine in $3.7 \%$ of the population, the maxillary first premolar tooth in $32.6 \%$, the maxillary second premolar tooth in $37.4 \%$, the maxillary first molar tooth in $25.3 \%$, and the maxillary second molar tooth in $1.1 \%$.

\section{Conclusions}

With the limitations of the study, the following conclusions can be drawn:

1. In the young Turkish population, the most common ST in both genders is the commissural smile, and the least common ST is the complex smile. 
2. The most common $\mathrm{SL}$ is the medium $\mathrm{SL}$, and the least common $\mathrm{SL}$ is the high $\mathrm{SL}$, and it varies by gender.

3. While the most common incisal line type is the convex line in both genders, the least common incisal line type is the reverse incisal line.

4. While the last visible maxillary tooth during the social smile is mostly the maxillary second premolar tooth, it is the maxillary second molar tooth at least.

Ethical Approval: Ethics committee approval was received for this study from Ataturk University.

Informed Consent: Written informed consent was obtained from all the patients who participated in this study.

Peer-review: Externally peer-reviewed.

Author Contributions: Conception -M.K.; Design - H.Ö.; Supervision - H.Ö., F.B.; Materials-H.Ö.; Data Collection and/or Processing-M.K.; Analysis and/or Interpretation - M.K., F.B.; Literature Review - F.B.; Writer - M.K., H.Ö.; Critical Review-H.Ö., F.B.

Conflict of Interest: No conflict of interest was declared by the authors.

Financial Disclosure: The authors declared that this study has received no financial support.

\section{References}

1. Morley J, Eubank J. Macroesthetic elements of smile design. J Am Dent Assoc 2001;132(1):39-45. [Crossref]

2. Gill DS, Naini FB, Tredwin CJ. Smile aesthetics. Dent Update 2007; 34(3):152-4, 157-8. [Crossref]

3. Sharma PK, Sharma P. Dental smile esthetics: The assessment and creation of the ideal smile. Seminars in Orthodontics 2012;18(3):193-201. [Crossref]

4. McLaren EA, Garber DA, Figueira J. The photoshop smilee design technique (part 1): digital dental photography. Compend Contin Educ Dent 2013;34(10):772, 774, 776 passim.

5. McLaren EA, Rifkin R. Macroesthetics: facial and dentofacial analysis. J Calif Dent Assoc 2002;30(11):839-46.

6. McLaren EA, Tran Cao P. Smile analysis and esthetic design: "in the zone". Inside Dent. 2009;5(7):46-8.

7. Philips E. The classification of smile patterns. J Can Dent Assoc 1999;65(6):252-4.

8. Ackerman JL, Ackerman MB, Brensinger CM, Landis JR. A morphometric analysis of the posed smile. Clin Orthod Res 1998;1(1):2-11. [Crossref]

9. Kulak Ozkan Y, Akoglu Vanlıglu B. Smile design in esthetic restoration. İstanbul: Vestiyer Pub. Group; 2014.

10. Garber DA, Salama MA. The aesthetic smile: diagnosis and treatment. Periodontol 2000 1996;11:18-28. [Crossref]

11. Sarver DM. The importance of incisor positioning in the esthetic smile: the smile arc. Am J Orthod Dentofacial Orthop 2001;120(2):98-111. [Crossref]

12. Sarver DM, Ackerman MB. Dynamic smile visualization and quantification: Part 2. Smile analysis and treatment strategies. Am J Orthod Dentofacial Orthop 2003;124(2):116-27. [Crossref]

13. Rubin LR. The anatomy of a smile: its importance in the treatment of facial paralysis. Plast Reconstr Surg 1974;53(4):384-7. [Crossref]

14. Al-Johany SS, Alqahtani AS, Alqahtani FY, Alzahrani AH. Evaluation of different esthetic smile criteria. Int J Prosthodont 2011;24(1):64-70.

15. Yoon ME, Jin TH, Dong JK. A study on the smile in Korean youth. J Korean Acad Prosthodont 1992;30(2):259-71.

16. AlQahtani NA, Haralur SB, AlMaqbol M, AlMufarrij AJ, Al Dera AA, Al-Qarni M. Distribution of smile line, gingival angle and tooth shape among the Saudi Arabian subpopulation and their association with gingival biotype. J Int Soc Prev Community Dent 2016;6(Suppl 1):53-8. [Crossref] 